Casos Clínicos

Arch. Esp. Urol., 58, 10 (1.061-1.064), 2005

\section{HEMATOSPERMIA Y QUISTE DEL CONDUCTO MÜLLERIANO}

\author{
Nuria Rodríguez García, Inmaculada Fernández \\ González, Carlos Pascual Mateo, Gino Espinales \\ Castro, Ana Ma García Tello y Antonio Berenguer \\ Sánchez.
}

Servicio de Urología. Hospital Univeritario de Getafe. Madrid. España.

Resumen.- OBJETIVO: presentación de un caso de quiste de utrículo tratado endoscópicamente.

MÉTODO: describimos el caso de un varón de mediana edad que consulta por hematospermia monosintomática de larga evolución. Al tacto rectal presentaba una próstata adenomatosa no sospechosa. Se practicó ecografía transrectal que revela quiste de utrículo complicado.

RESULTADOS: Se realizó marsupialización endoscópica del quiste, consiguiendo remisión total de la hematospermia.

CONCLUSIONES: Se debe realizar ecografía transrectal a todos los pacientes que consultan por hematospermia de larga evolución, indicando la marsupialización endoscópica del quiste del conducto mülleriano en aquellos pacientes sintomáticos y con quistes complejos observados en la exploración ecográfica, obteniendo un importante porcentaje de cura o mejoría en este grupo de pacientes sin inducir ningún efecto colateral secundario.

Palabras clave: Hematospermia. Conducto mülleriano. Quiste de utrículo.

Nuria Rodríguez García

Servicio de Urología

Hospital de Getafe

Ctra. de Toledo km. 12,500

28905 Getafe. Madrid. (España)

Trabajo recibido: 28 de marzo 2005
Summary.- OBJECTIVES: To report one case of utriculum cyst treated endoscopically.

METHODS: We describe the case of a mid-age male patient consulting for long-term monosymptomatic hemospermia. Rectal digital examination revealed a non suspicious adenomatous prostate. Transrectal ultrasound showed a complicated utriculum cyst.

RESULTS: Endoscopical marsupialization of the cyst was performed with complete remission of hemospermia.

CONCLUSIONS: Transrectal ultrasound should be performed in all patients presenting with long-term hemospermia. Endoscopical marsupialization of the mullerian duct cyst is indicated in symptomatic patients or those with complex cysts on ultrasound, offering a high cure/improvement rate in this group of patients without secondary effects.

\section{Keywords: Hemospermia. Mullerian duct.}

Utriculum cyst.

\section{INTRODUCCIÓN}

El uso generalizado de la ecografía transrectal para evaluar diferentes patologías urológicas, ha proporcionado la oportunidad de documentar distintas anomalías que pueden ser responsables de los síntomas de presentación o no tener ningún efecto en el paciente. El utrículo prostático habitualmente es una cavidad virtual y no visible en la ecografía transrectal excepto cuando está aumentado. Los quistes de utrículo se suelen visualizar de forma incidental cuando se realiza la ecografía transrectal por otras razones como son: sintomatología relacionada con el tracto urinario inferior en la hiperplasia benigna de próstata, dolor, hematospermia, infección del tracto urinario inferior e infertilidad (1). Se describe en este artículo el diagnóstico y tratamiento de un quiste de utrículo complicado en un varón que consulta por hematospermia

\section{CASO CLÍNICO}

Varón de 55 años de edad que consulta por hematospermia de 1 año de evolución. No refiere ningún antecedente médico de interés. En la exploración física el tacto rectal es normal, con una próstata adenomatosa volumen mediano. La analítica de sangre, el cultivo de orina y semen y el valor del PSA son normales. En la ecografía transrectal se observa un quiste del conducto mülleriano, localizado en línea media, con una pared fina e hiperecoica y con una masa ecogénica en su in- 
terior con un diámetro de 0,5 cm. (Figura 1). Se decide la realización de una exploración endoscópica transuretral de la cavidad. Con el resector convencional y con el cuchillete de Collins se procede al destechamiento de la cavidad cranealmente al verumontanum y con la bola se realiza hemostasia de los bordes de la misma; en el interior de la cavidad no se visualiza ninguna masa en su interior y solamente se observan dos litiasis de pequeño tamaño. La inspección endoscópica del quiste permite la visualización de los conductos eyaculadores (Figura 2). Al año de seguimiento el paciente está asintomático y no ha vuelto a presentar hematospermia. En la ecografía transrectal realizada en el seguimiento se observa la desaparición del quiste.

\section{DISCUSIÓN}

La incidencia de utrículos prostáticos dilatados oscila entre el 11 al $14 \%$ en presencia de hipospadias o estados intersexuales, siendo mayor al 50\% cuando existe un hipopadias perineal (2). Generalmente se asocian con anormalidades en los genitales y se diagnostican en pacientes jóvenes; son de gran tamaño y de forma vesicular o tubular; frecuentemente se comunican con la uretra prostática y han sido denominados quistes de utrículo o vagina masculinus (3).

En contraste, los quistes del conducto mülleriano son redondos, no comunican con la uretra prostática y son diagnosticados en adultos con genitales externos normales. La incidencia oscila entre el $5 \%$, en varones a los que se les practica una ecografía transrectal para el diagnóstico del adenocarcinoma prostático, al 17\% en varones infértiles en los que el análisis de semen arroja la sospecha de posible obstrucción de los conductos eyaculadores (4). De modo que no son raros y puesto que están presentes frecuentemente, pero latentes en pacientes asintomáticos o poco sintomáticos, resulta un problema para el urólogo cuando se desarrollan los síntomas.

Cuando son sintomáticos existen diferentes formas de presentación clínica siendo las más frecuentes la hematospermia, que aparece en el $40 \%$ de los casos, sola o asociada a dolor testicular o pélvico-perineal y/o infección del tracto urinario inferior y los síntomas irritativos vesicales y/o uretrales en el $25 \%$ de los casos (micción dolorosa; aumento de frecuencia miccional, disuria, urgencia y sensación de vaciamiento incompleto vesical (5).

Ecográficamente los quistes del conducto mülleriano aparecen como estructuras redondas, anecoicas, sin pared hiperecoica, no asociados a calcificaciones y asociados a ampollas deferenciales y vesículas seminales normales. Se consideran quistes del conducto mülleriano complejos o complicados aquellos que reunen una o más de las siguientes características: crecimiento paramedial; contenido ecogénico; delineación hiperecoica o adelgazamiento de la pared; focos hiperecoicos o calcificación a nivel del verumontanum, conductos eyaculadores o vesículas seminales; asociado a ausencia, asimetría o dilatación de la ampolla deferencial o vesículas semi-

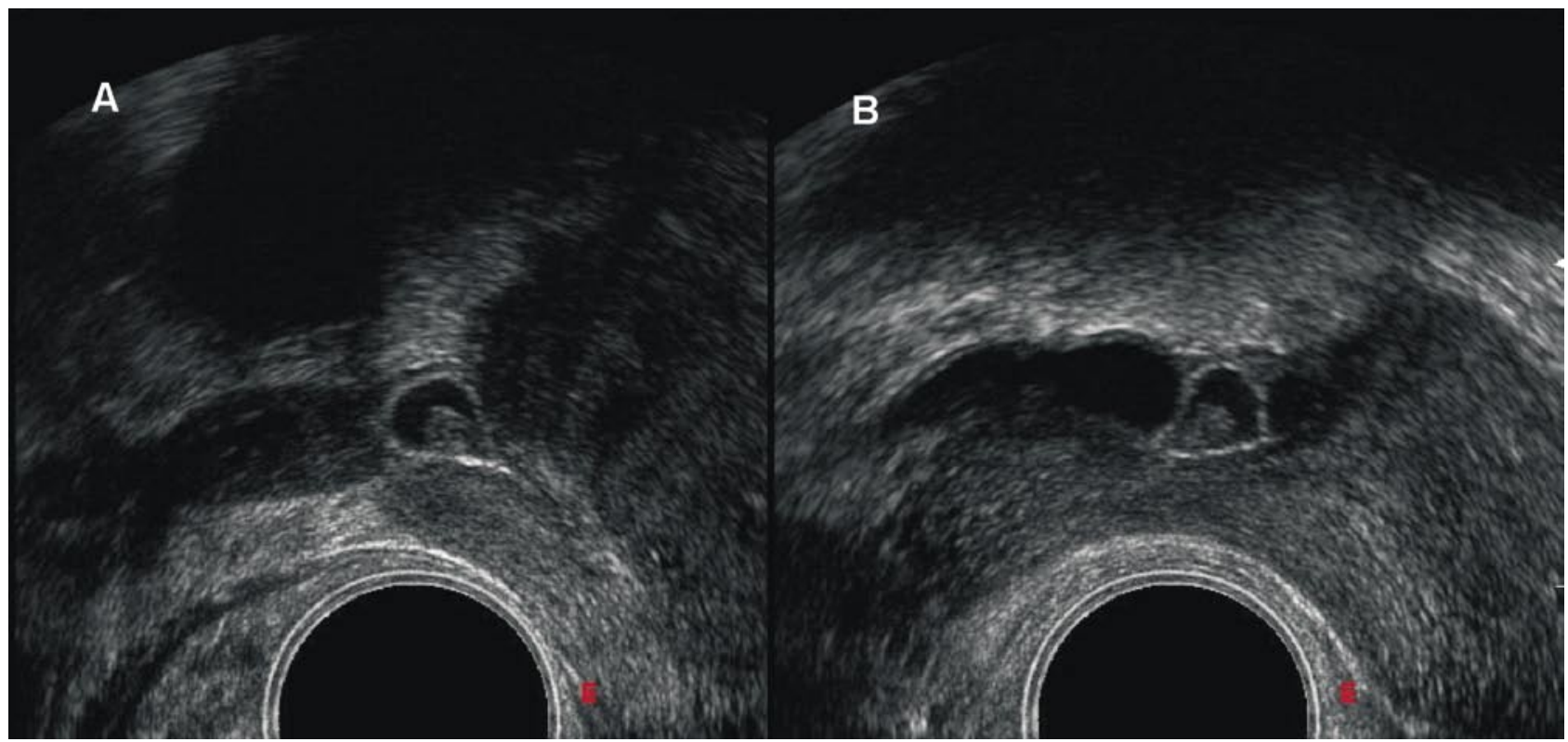

FIGURA 1. A) Ecografía transrectal del quiste del conducto mülleriano, sección longitudinal, donde se observa un quiste con pared hiperecoica y con contenido ecogénico en su interior;

B) Ecografía transrectal, sección transversal, el quiste se sitúa en la línea media. 
nales; presencia de estructuras quísticas adicionales y aquellos divididos por un septo medio (5).

El quiste del conducto mülleriano no complicado puede tratarse mediante la aspiración de su contenido guiados con ecografía transerctal (6-8). Sin embargo en aquellos quistes complejos es recomendable su amplia marsupialización por vía endoscópica con fines diagnósticos y/o terapéuticos, siendo infrecuente la existencia posterior de reflujo de orina en la vía seminal $(9,10)$.

La biopsia rutinaria de la pared del quiste no está indicada ya que el diagnóstico anatomo-patológico suele ser normal a pesar de los hallazgos ecográficos o anormalidades endoscópicas observadas. Los resultados del cultivo y biopsia de la pared del quiste en aquellas

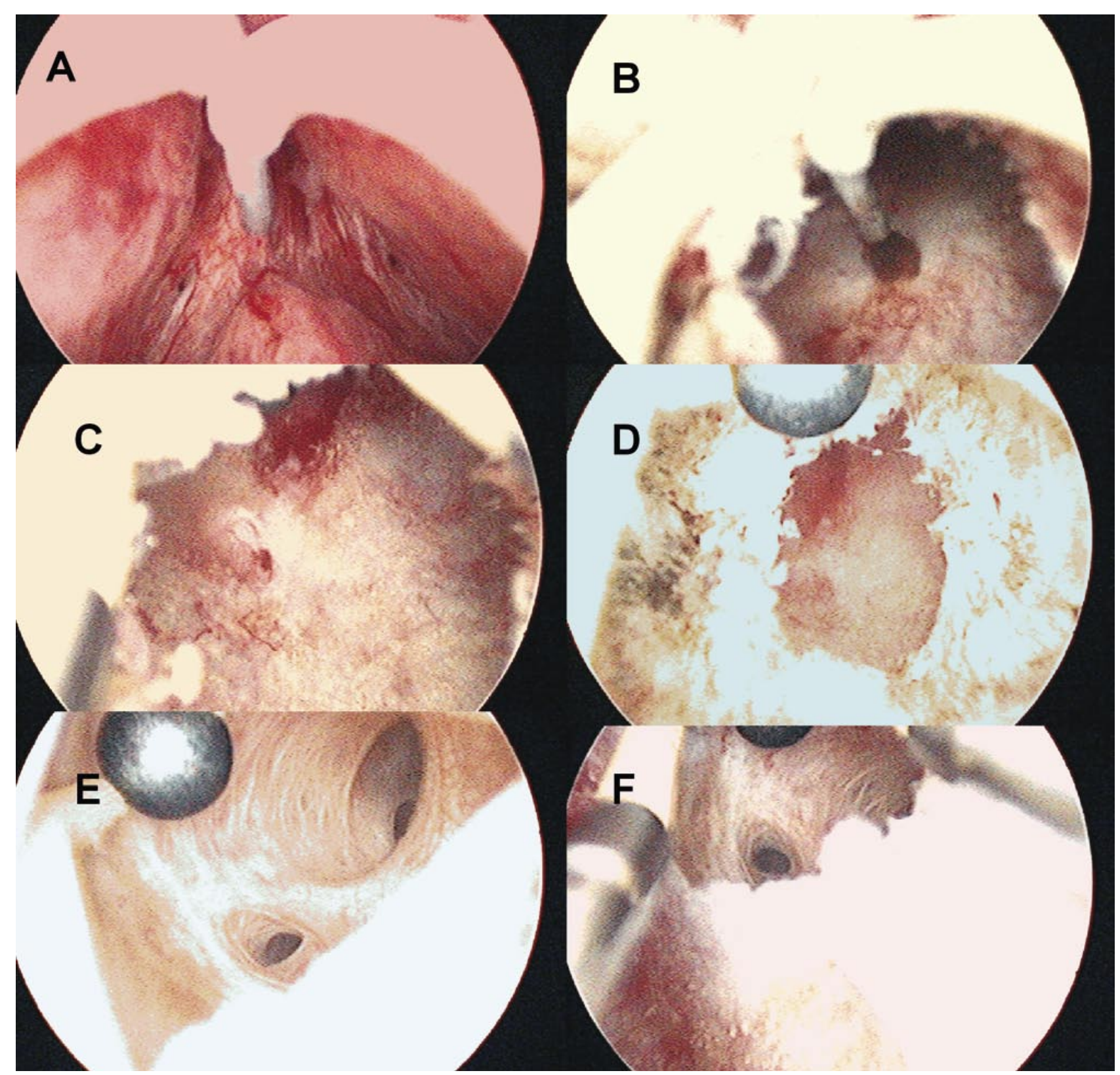

FIGURA 2. A) Visión endoscópica previa al destechamiento del quiste con el cuchillete de Collins;

B) Una vez que se destecha la cavidad se observa un pequeño cálculo en su interior;

C) No se observa ninguna masa sólida en su interior, consecuentemente la imagen ecográfica se correspondería con un coágulo;

D) Coagulación con la bola de los bordes de la cavidad con fines hemostáticos;

E) Conducto eyaculador izquierdo que se comunica con el quiste;

F) Conducto eyaculador derecho. 
cavidades que aparecen delineadas por una pared hiperecoica no aportan ninguna información adicional. Tampoco existe explicación para la alta incidencia de hematospermia en estos pacientes, pero existe evidencia que cede con la masurpialización del quiste (5).

\section{CONCLUSIONES}

Se debe realizar ecografía transrectal a todos los pacientes que consultan por hematospermia de larga evolución, indicando la marsupialización endoscópica del quiste del conducto mülleriano en aquellos pacientes sintomáticos y con quistes complejos observados en la exploración ecográfica, obteniendo un importante porcentaje de curación o mejoría en este grupo de pacientes sin inducir ningún efecto colateral secundario.

\section{BIBLIOGRAFÍA y LECTURAS RECOMENDADAS ( ${ }^{*}$ lectura de interés $y^{* *}$ lectura fundamental)}

1. COPPENS, L.: "Diagnosis and treatment of obstructive seminal vesicle pathology". Acta Urol. Belg., 65: 11, 1997.

2. DEVINE, C.J.; GONZÁLEZ-SERVA, L.; STECKER, J.F. y cols.: "Utricular configuration in hypospadias and intersex". J. Urol., 123: 407, 1980.

3. RITCHEY, M.L.; BENSON, R.C.; KRAMER, S.A. y cols.: "Management of müllerian duct remnants in the male patient". J. Urol., 140: 795, 1988.

4. KIM, E.D.; ONEL, E.; HONIG, S.C. y cols.: "The prevalence of cystic abnormalities of the prostate involving the ejaculatory ducts as detected by transrectal ultrasound". Int. Urol. Nephrol., 29: 647, 1997.

5. COPPENS, L.; BONNET, P.; ANDRIANNE, R. y cols.: "Adult mullerian duct or utricle cyst: clinical significance and therapeutic management of $65 \mathrm{ca}-$ ses". J. Urol., 167: 1740, 2002.

6. ARAGONA, F.; SPINELLI, C.; CAMPATELLI, A. y cols.: "Urinary retention due to a müllerian duct cyst: role of ultrasonically guided fine needle aspiration in the diagnosis and treatment". J. Urol., 134: 364, 1985.

7. FELDERMAN, T.; SCHELLHAMMER, P.F.; DEVINE, C.J. y cols.: "Müllerian duct cysts: conservative management". Urology, 29: 31, 1987.

8. STRICKER, H.J.; KUNIN, J.R.; FAERBER, G.J.: "Congenital prostatic cyst causing ejaculatory duct obstruction: management by transrectal cyst aspiration". J. Urol., 149: 1141, 1993.

9. WEINTRAUB, C.M.: "Transurethral drainage of the seminal tract for obstruction, infection and infertility". Br. J. Urol., 52: 220, 1980.

10. VAN POPPEL, H.; VEREECKEN, R.; DE GEETER, P. y cols.: "Hemospermia owing to utricular cyst: embryological summary and surgical review". J. Urol., 129: 608, 1983.
Casos Clínicos

Arch. Esp. Urol., 58, 10 (1.064-1.068), 2005

\section{INFECCIÓN POR HTLV POST-TRASPLANTE RENAL.}

Ainara Villafruela Mateos, Antón Arruza Echevarría, Jesús Martín Bazaco, lgor Azurmendi Arin, José Antonio Zabala Egurrola y Carlos Pertusa Peña.

Servicio de Urología. Hospital de Cruces. Baracaldo. Vizcaya. España.

Resumen.- OBJECTIVOS: Presentamos los 2 primeros casos de infección por HTLV-l tras trasplante renal ocurridos en nuestro país y su evolución (1998).

MÉTODOS: Se describen los casos de 2 pacientes trasplantados que han desarrollado una mielopatía subaguda, la Paraparesia Espástica Tropical, secundaria a infección por HTLV-l y se revisa su evolución.

RESULTADOS: En ambos casos, en el momento actual, presentan gran incapacidad, siendo, en concreto un paciente, dependiente para la vida diaria.

CONCLUSIONES: Creemos necesaria la detección de HTLV-I, en todos los donantes, mediante técnicas indirectas (enzimoinmunoensayo, ELISA) o directas (PCR) dada la evolución tórpida de la infección en el paciente trasplantado por un lado y por otro, por la creciente población inmigrante en nuestro país que conlleva un aumento de portadores asintomáticos del virus.

Palabras clave: HTLV-I. Infección. Trasplante renal.

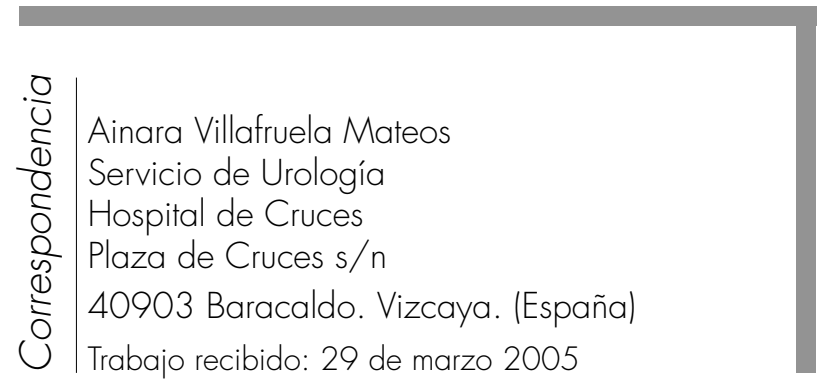

